REVISTA ECONOMÍA

Vol. 69, N. ${ }^{\circ}$ Io9 (mayo), I29-I48

\title{
¿LA COMERCIALIZACIÓN ASOCIATIVA DE LOS PEQUEÑOS AGRICULTORES MEJORA LOS INGRESOS? EVIDENCIA DE LOS AGRICULTORES DE PAPA EN EL ECUADOR
}

\author{
NANCY MEDINA CARRANCO \\ Universidad Central del Ecuador
}

Recepción manuscrito: 6 de enero de 2017

Aceptación versión final: 15 de abril de 2017

\begin{abstract}
RESUMEN El presente artículo analiza los impactos del programa Plataformas de Concertación y CONPAPA, orientado a mejorar el bienestar de los pequeños productores de papa en la sierra central del Ecuador. Se trata de determinar si el programa logró los impactos deseados y si éstos fueron sostenibles para los productores de la provincia de Tungurahua. Se utiliza un diseño cuasiexperimental con un grupo de control y otro de tratamiento que incluyó a los socios de CONPAPA actuales y a los que fueron hace cuatro años. Los resultados sugieren un impacto positivo significativo en los rendimientos, los beneficios brutos y la aplicación de ciertas prácticas culturales.
\end{abstract}

PALABRAS CLAVE Mercado, evaluación de impacto, pequeños productores de papa, bienestar.

ABSTRACT This article analyzes the impacts of the Plataformas de Concertación y CONPAPA program, aimed at improving the welfare of small potato producers in the central sierra of Ecuador. It is about to determine if the program achieved the desired impacts and whether these were sustainable for the producers of the province of Tungurahua. A quasi-experimental design was used with a control group and a treatment group that included the current CONPAPA partners and those who they were four years ago. The results suggest a significant positive impact on yields, gross profits and the application of certain crop practices.

KEYWORDS Market, impact assessment, small potato producers, welfare.

JEL CODES D63, I38, $\mathrm{P}_{32}, \mathrm{D}_{72}$.

\section{INTRODUCCIÓN}

En el Ecuador es de gran importancia considerar al sector agrícola para impulsar una estrategia de reducción de la pobreza, ya que en el sector rural vive el 37,23\% de la población total, de los cuales el $57,73 \%$ es pobre, siendo el $41,69 \%$ de la población clasificada como pobre y el 16,04\% como pobres extremos; mientras que en el área urbana, $16,3 \%$ son considerados pobres y 4,2\% como extremos pobres (INEC, 2014). Adicionalmente, el 72,7\% de la población del área rural trabajan en la rama de actividad denominada agricultura, caza y silvicultura, de acuerdo a la Encuesta Nacional de Empleo y Desempleo (ENEMDU) realizada en diciembre del 2014 por el INEC. 
La zona de planificación 3 y 5, donde se ubican las provincias de Chimborazo, Tungurahua, Cotopaxi y Bolívar, zonas de intervención del programa objeto de evaluación, muestran un alto nivel de pobreza y desigualdad territorial por necesidades básicas insatisfechas (NBI); registrando el 72,6\%, en promedio, superior al nacional 61,3\% (SENPLADES, 2010). Las áreas con los porcentajes más altos de pobreza por NBI en personas en esta zona son: Colta-Guamote (Chimborazo) con 94,4\%; Sigchos (Cotopaxi) con 93,7\%; Colta, Mocha y Tisaleo (Tungurahua) con el 76\% (SENPLADES, 2015); y la provincia de Bolívar que registra el 92,5\%, en promedio. Se constata que los niveles más altos de NBI se registran en zonas rurales y sobre todo afectan a la población femenina e indígena.

El sector agrícola es estratégico para superar los problemas de pobreza, es así que tanto en la década de los noventa como en el año 2008, el Banco Mundial comprobó empíricamente que el crecimiento agrícola es capaz de reducir la pobreza en cualquier país. Es así que se dice que, según estimaciones realizadas en diversos países, el crecimiento del pIB agrícola es «al menos el doble de eficaz en reducir la pobreza que el crecimiento del PIB de otros sectores» (Banco Mundial, 2008, p. 24), incluso es más eficaz que el crecimiento industrial para reducir la pobreza urbana (Vollrath, 1994). El crecimiento agrícola contribuye al desarrollo económico debido a sus efectos multiplicadores sobre la economía interna. Con el reconocimiento del rol del sector agrícola para el desarrollo y alivio de la pobreza, se dieron cambios sustantivos de la concepción de la política agrícola en todo el mundo y con ello de la cooperación internacional para la agricultura.

A inicios de los años 2000, la mayoría de programas de la cooperación internacional apuntaron a la vinculación más efectiva de los agricultores al mercado, al incremento de productividad de cultivos y la comercialización asociativa; en este contexto se ubica la intervención del programa de Plataformas de Concertación y CONPAPA, que es el objeto de esta investigación que busca determinar su impacto en el bienestar de los pequeños productores de papa en la provincia de Tungurahua.

La intervención del programa de Plataformas de Concertación y conPAPA estaba dirigida a reducir la pobreza, por lo cual intervino en las provincias de Chimborazo, Tungurahua, Cotopaxi y Bolívar - parte Sierra-, áreas que registran los niveles más altos de NBI. Fue una estrategia que implementó acciones en varios componentes, los cuales permitieron un proceso participativo, en particular de los pequeños productores de papa, cuya organización, Consorcio de Pequeños Productores de Papa (CONPAPA), fue constituida en el marco del programa y todavía está operativa. Es así que la intervención incluyó desde las escuelas de campo (ECA) como un medio de capacitación y una forma de motivar a la participación, donde se procuró el aprendizaje del manejo del cultivo orientado a la racionalización del uso de agroquímicos y de protecciones para su aplicación, hasta la comercialización asociativa, donde los productores se organizaron para vender en conjunto. Para esto, planificaron la producción y produjeron las variedades de papa demandadas por el mercado, mejoraron la calidad del producto y negociaron en conjunto para tener mejores condiciones de venta.

La intervención de referencia es la continuación de un programa que inició en la década de los años noventa -Fortalecimiento de la Investigación y Producción de Papa (FORTIPAPA) donde el énfasis fue en aspectos de la producción. Esta investigación se refiere a la segunda parte del programa que inició en el año 2003 y cerró en el año 2009 con la salida de la Cooperación 
Suiza para el Desarrollo (COSUDE), donde lo importante fue la construcción de una nueva institucionalidad. La intervención tuvo un enfoque diferente, pues empezó considerando las necesidades reales de las comunidades — enfoque de demanda-, las cuales eran identificadas en forma participativa con los beneficiarios del programa. También se vio que era necesario ejecutar el programa con comunidades organizadas, lo cual era beneficioso para impulsar la asociatividad como estrategia frente a los retos de inicios de los años 2000, donde se vivía un proceso de globalización que hacía notar que era mejor actuar en conjunto antes que de manera individual, por ejemplo, para comercializar porque esto permitía tener mejores condiciones de venta.

Bajo estos lineamientos se plantearon el Fortalecimiento del ConPaPA, Plataformas de Concertación y CONPAPA, (FORTICONPAPA), que consistió en tres etapas bien identificadas en el período 2003 al 2009: i) la construcción de una nueva institucionalidad -2003 a 2005- en la cual intervenían los GAD, ${ }^{1}$ la cooperación internacional, instituciones del gobierno central como el MAGAP y otros actores del territorio; ii) el funcionamiento de las plataformas y del CONPAPA — 2006 a 2007-; y iii) el protagonismo del CONPAPA — 2008 a 2009- (Reinoso $e t$ al., 2009). La intervención tuvo varios componentes: investigación, institucionalidad, semillas, comercialización y capacitación (Reinoso et al., 2009).

En el componente de investigación se incluyeron estudios para combatir plagas y enfermedades del cultivo de la papa y el desarrollo de nuevas variedades que se seleccionaban en función del potencial en el mercado para consumo en fresco y procesamiento, como fue el caso de la variedad INIAP-Fripapa resistente a la precocidad. A esto se sumaron los estudios para manejo poscosecha, almacenamiento, prácticas de cultivo y uso de insumos amigables con el medio ambiente (Reinoso et al., 2009).

En la parte institucional se trataba de impulsar la participación directa de los pequeños productores de papa y actores locales. Se inició con las plataformas como espacios de concertación que reunían a diferentes actores del sector privado y público, incluyendo a las ONG nacionales e internacionales que aportaban con financiamiento, conocimiento e investigación, $\mathrm{u}$ organizando a los productores. Lo importante fue que se llegó al protagonismo de los pequeños productores a través del CONPAPA (Reinoso et al., 2009).

En el componente referido a semillas, su manejo evolucionó desde un enfoque de seguridad alimentaria a uno como insumo de mercado a fin de reducir los costos de producción, esto impulsó la conformación de una red de semilleristas, quienes producían semillas para vender tanto a los socios de CONPAPA como a otros productores (Reinoso et al., 2009). En 2013, el CONPAPA continúa vendiendo semillas que producen un grupo de 15 semilleristas.

La comercialización asociativa se constituyó en un eje aglutinador importante el cual empezó para proveer de la variedad fripapa para hojuelas a la empresa Fritolay. Se formaron empresas comercializadoras en tres provincias del programa —Chimborazo, Tungurahua y Bolívar-, las cuales fueron transferidas poco a poco a los pequeños productores, aunque siempre hubo un gerente que las manejaba, tanto para negociar contratos de venta como para planificar la producción (Reinoso et al., 2009). La comercialización asociativa permite que los pequeños productores logren mejores condiciones de venta para todos, lo cual se lo puede hacer gracias a que al tener una oferta importante, derivada de la suma de la producción de los participantes, tienen poder de negociación. 
La capacitación tuvo gran importancia en la conformación del tejido social que luego sería sostén de las Plataformas, CONPAPA y la red de semilleristas (Reinoso et al., 2009). Se aplicó un enfoque sistémico, donde los participantes con mayor presencia eran los pequeños productores y se tendía a un equilibrio entre la teoría y la práctica (Andrade Piedra et al., 2013). Las capacitaciones se hicieron con base en la metodología de las Escuelas de Campo (ECA) y la creación de los cdc — capacitación de capacitadores- (Reinoso et al., 2009).

En este contexto, la pregunta de investigación sería: ¿Una intervención con enfoque de cadena productiva que se consolida con la comercialización asociativa de pequeños productores de papa logra impactos sostenibles que permiten mejorar el bienestar de los beneficiarios, reduciendo la pobreza?

La investigación que se presenta en este artículo pretende dar respuesta a esta pregunta considerando únicamente a la provincia de Tungurahua. Lo interesante y el valor agregado de esta investigación consiste en que evalúa los impactos prácticamente cuando el CONPAPA dejó de tener apoyo financiero, a los cuatro años de estar caminando solo. Adicionalmente, para evaluar el impacto se utiliza una muestra para el grupo de tratamiento que incluye no solo a los que todavía eran socios del consorcio en el año 2013, sino a un grupo de los que dejaron de ser socios, lo cual permite identificar los impactos de mejor forma y su sostenibilidad.

El documento está organizado en 9 secciones: en la primera se hace una introducción. La segunda sección contiene la revisión de la literatura; mientras que la tercera hace una descripción del programa a ser evaluado. La cuarta sección presenta el diseño metodológico de la investigación, en tanto que la quinta sección describe el proceso de recolección y análisis de datos. En la sexta se especifica el enfoque empírico y, a continuación, se discuten los resultados de la investigación. Finalmente se presentan las conclusiones y las referencias bibliográficas.

\section{REVISION DE LA LITERATURA}

Dado el tipo de intervención que se analiza, donde se incluyen acciones desde la producción hasta la comercialización, esta investigación está en el contexto de cadena de valor de la papa. Además se relaciona con la asociatividad en donde prima la visión de «suerte común» o interés de todos, es decir, se incluye la solidaridad en la economía de la organización, lo cual según Luis Razeto (1999) corresponde a economía solidaria.

A partir del año 2006, han crecido significativamente las iniciativas de desarrollo de cadenas de valor, las que se reconocen como un enfoque apropiado para promover el desarrollo en zonas rurales. Adicionalmente, la fuerte subida de los precios de los alimentos en el mundo entre los años 2008 y 2009 motivó a muchas empresas privadas a buscar fuentes sostenibles de materias primas, y a ampliar sus servicios a los consumidores de los países en vías de desarrollo a fin de desarrollar una ventaja comparativa comercial a largo plazo (Camagni y Kherallah, 2016).

El enfoque de cadenas de valor se basa en una visión integral de todas las actividades necesarias para producir un bien y llegar al mercado, esto es desde la provisión de insumos para la producción hasta que llega al consumidor final, siendo el mercado «el motor básico» de una cadena de valor (Camagni y Kherallah, 2016). Esto significa que los productores no tienen motivación alguna sin la demanda del mercado de los consumidores. Ahora, que la mejor 
forma de acceder a los mercados por parte de los pequeños productores es en forma colectiva o asociativa, a fin de disminuir los altos costos de transacción. Más aún si están organizados pueden también beneficiarse de las economías de escala en los mercados de insumos o de productos. El aumento del poder de negociación de los pequeños agricultores en este tipo de arreglo, puede ayudar a asegurar que los beneficios sean compartidos (Banco Mundial, 2008).

Por otro lado, los pequeños productores asociados pueden acceder a mercados de alto valor, lo que les abre nuevas oportunidades para que se promueva la innovación en la cadena de valor, las mismas que para ser aprovechadas necesita que se asocien el sector público, el privado, los agricultores y la sociedad civil a fin de financiar, desarrollar y adaptar las innovaciones (Banco Mundial, 2008).

En este contexto, a fin de fortalecer la aplicación del enfoque de cadena de valor, el Instituto Nacional de Investigaciones Agripecuarias (INIAP), con el apoyo de la cosUdE, impulsó las plataformas de concertación, novedosa institucionalidad con la que inició el programa, como espacios de «alianzas concertadas entre los actores locales, públicos y privados, para desarrollar acciones en componentes como: comercialización, organización, producción e investigación» (Reinoso et al., 2009). Esto permitió lograr el apoyo de diversos actores locales en los diferentes eslabones de la cadena y de otros que podría influir directa e indirectamente, lo que iba consolidando la forma de llegar a una institucionalidad en la que los pequeños productores sean los protagonistas. ${ }^{2}$

Para verificar los impactos del programa se procedió a realizar una evaluación de impacto. Se puede encontrar una vasta literatura que examina el impacto que tiene la asociatividad en la comercialización sobre el precio de venta de los productos agrícolas, los niveles de ingresos, los costos y la rentabilidad de las fincas campesinas; así como sobre los problemas de la sostenibilidad temporal y económica de este tipo de instituciones, de los programas caracterizados por vincular a los productores al mercado, buscar el incremento de productividad de cultivos e impulsar la comercialización asociativa. Comúnmente la evaluación de impactos de este tipo de programas es de naturaleza ex post, debido a que no se incluye en la formulación del proyecto, lo que se explica por el hecho que, en la mayoría de los casos, los experimentos son difíciles de llevarlos adelante por una serie de razones, incluyendo costos y aspectos prácticos (Cavatassi et al., 2011). A pesar de que los programas y proyectos revisados tienen como sujeto a los pequeños productores, los resultados de las intervenciones se diferencian y, a menudo, las conclusiones son contradictorias.

Fischer y Qaim (2012) demostraron que el incremento en los ingresos para los pequeños productores de banano en Kenia se relacionaba con la asociatividad. Encontraron efectos positivos en los ingresos de los miembros activos del grupo; aunque las ventajas de los precios de la comercialización colectiva fueron pequeños y las potencialidades del mercado de alto valor $^{3}$ aún no se habían aprovechado. El objetivo principal de estos autores fue identificar los factores que determinan la asociatividad considerando a los costos - aportes, transporte para entrega de productos, tiempo de actividades conjuntas - y a los beneficios - mejor acceso a los mercados de insumos y productos, incluida la tecnología y la información- de la misma. Ellos aplicaron el método de emparejamiento Propensity Score Matching (PSM) para modelar en dos etapas el efecto de la asociatividad. 
Bachke (2009) señala que en Mozambique la membresía en una organización permite un impacto positivo en los ingresos de los pequeños productores agrícolas, lo que conduce a la autora a sostener que la organización campesina puede ser considerada como una herramienta para la reducción de la pobreza, a pesar de no contar con resultados que expliquen el medio del incremento notado en los ingresos: precios, productividad o ambos. Ella utilizó datos de panel y el método de diferencias en diferencias.

Bernard et al. (2008) encuentran a través del método de PSM que la acción cooperativa en zonas rurales de Etiopía genera un precio de venta más alto para los miembros de la cooperativa. Sin embargo, el incremento de precios de venta no necesariamente conduce a un comportamiento más orientado al mercado. La magnitud de la producción comercializada responde a la proporción del autoconsumo en la producción total de los pequeños productores agrícolas, más que a la variación de los precios.

Sikwela y Mushunje (2013) identificaron el efecto causal de diferentes servicios de apoyo o estructuras - arreglos de gobernabilidad-, planteando la hipótesis que tales intervenciones tenían un efecto sobre los activos y las capacidades de los agricultores para acceder a los mercados y lograr mejores pagos, proporcionando conocimientos técnicos, información, capacitación, servicios de asesoramiento y servicios dentro y fuera de las infraestructuras agrícolas. Para evitar sesgos de selección no aleatoria y la autoselección, no se consideraron a los productores individuales cercanos a las zonas de los productores que recibían apoyo; $y$, para identificar los impactos se utilizaron el método de emparejamiento PSM y un modelo Tobit. Los hallazgos de esta investigación muestran que los programas de apoyo a los agricultores y las actividades de comercialización colectiva, tiene un impacto significativo y positivo en el bienestar de los pequeños agricultores.

Wainaina et al. (2012) encuentran un impacto positivo en los ingresos de productores en Kenia, cuando los agricultores suscriben un contrato de producción, para lo cual utilizó el método PSM. Godtland et al. (2004) muestran que las escuelas de campo (ECA) son efectivas en impartir el conocimiento sobre manejo integrado de plagas (MIP) de la papa a los agricultores de Perú. Mientras que un mejor conocimiento de las prácticas de mIP tiene el potencial de mejorar significativamente la productividad en la producción de papa. Ellos utilizaron tanto para formar los grupos de control y tratamiento, como para estimar el impacto el método de PSM.

Berdegué Sacristán (2001), al examinar el impacto de las Empresas Asociativas Campesinas (EAC), promovidas por el gobierno chileno al principios de los noventa, argumenta que las EAC tienden a ser más exitosas cuando operan en los mercados que se caracterizan por altos costos de transacción, difícil acceso a la información sobre los precios, entradas condicionadas por la inversión, tecnología o conocimiento y gestión, y de productos altamente perecederos. No obstante, solo alrededor de una quinta parte de las EAC podrían sobrevivir si los programas gubernamentales se interrumpieran repentinamente, mientras que un $15 \%$ adicional probablemente podría consolidar su posición. Analizó las pequeñas explotaciones dedicadas a la producción de leche, y a los cultivos de papa y trigo por separado, utilizando el procedimiento de dos etapas de Heckman, el cual le permitía controlar el sesgo de selección de las estimaciones del impacto del programa.

En el Ecuador, el programa Plataformas de Concertación y conPAPA es un ejemplo de las intervenciones para impulsar a los pequeños productores de papa en la Sierra central del país. 
González-Flores et al. (2014) sostienen que el programa tuvo un impacto positivo sobre los rendimientos de cultivo de papa. Sin embargo, los participantes del programa mostraron una eficiencia técnica menor en relación a los del grupo de control, lo que puede encontrar su explicación por el hecho de que al momento de recolectar los datos, los beneficiarios aún se encontraban en la parte creciente de la curva de aprendizaje. Para estimar los impactos utilizaron el método PSM combinado con un modelo de Frontera de Producción Estocástica que corrige el sesgo de selección de la muestra en modelos no lineales, desagregaron el aumento del rendimiento atribuible al programa en el cambio tecnológico (Ст) y la eficiencia técnica (Ет).

Cavatassi et al. (2011) identifican amplios impactos positivos del programa para la mejora del bienestar de los pequeños productores de papa, expresados en variables de resultados como las utilidades brutas y la productividad de cultivos, para lo cual utilizaron varios métodos como Mínimos Cuadrados Ordinarios (MCO), PSM, PSM ponderado de Mínimos Cuadrados (WLS) y variables instrumentales (IV). Los autores no encontraron evidencias negativas del programa en relación al medio ambiente y a salud de los productores. A pesar de que Plataformas de Concertación y CONPAPA ya cuenta con dos evaluaciones de impacto, ambas fueron realizadas mientras el programa tuvo apoyo financiero externo. El reto que enfrenta la presente investigación consiste en comprobar la sostenibilidad de los impactos del programa en el tiempo, una vez retirada la asistencia externa.

\section{DESCRIPCIÓN DEL PROGRAMA}

El programa Plataformas de Concertación y CONPAPA pertenece a la segunda etapa del proyecto Fortalecimiento de la Investigación y Producción de Papa, FORTIPAPA, implementado por el INIAP y ejecutado en colaboración con el Centro Internacional de la Papa (CIP) y su Programa Colaborativo Papa Andina con el apoyo financiero de la Agencia Suiza para el Desarrollo y la Cooperación (COSUDE), como un instrumento de fortalecimiento de pequeños productores de papa en la Sierra Central del Ecuador (Reinoso et al., 2009).

La primera etapa (1992-2001) del programa se centró en la investigación participativa y la transferencia de tecnología, la segunda etapa (2001-2009) se caracterizó por un enfoque hacia el empoderamiento de los pequeños productores y pequeñas productoras en la cadena agroalimentaria de la papa, a partir de formación de la nueva institucionalidad de la papa (Crespo Coello et al., 2005). La reorientación del programa responde a la instauración de nuevos paradigmas en el desarrollo. Por un lado, ocurre en el período en el que surge y se posiciona con alta relevancia el enfoque de combate contra la pobreza, lo que implica la necesidad de priorizar estrategias para incrementar los ingresos de los pequeños productores y productoras. Por otro lado, surge con fuerza el concepto de desarrollo local desde abajo, donde los ciudadanos se convierten en protagonistas de su propio desarrollo, lo que implica la construcción de ciudadanía frente al deterioro de la institucionalidad del Estado central.

Bajo estas premisas, los años 2002-2004 son de montaje de una nueva institucionalidad de la papa. En el 2003 iniciaron su acción las Plataformas de Concertación y en 2004 se formó el Consorcio de Productores de Papa del Ecuador (CONPAPA). Reinoso et al. (2009, p. 15) considera que: 
El ConPaPA es el Consorcio de Productores de Papa del Ecuador, el cual tiene dos fines. Primero, comercializar en mejores condiciones la papa y articularse a cadenas de valor, para lo cual requiere de reglas de producción y comercialización que exija a sus socios estándares de calidad, cantidad y continuidad en la provisión. Segundo, posicionar a los productores de la papa y al mismo rubro en mejores condiciones dentro del entorno sociopolítico y económico local y nacional.

Por lo tanto, las plataformas y el CONPAPA constituyen espacios de alianza, agremiación y emprendimiento empresarial y comercial que tiene como objetivo empoderar y articular a los pequeños productores de papa de la Sierra central ecuatoriana con mercados y con cadenas de valor. Todo esto para mejorar los ingresos de las familias campesinas participantes en el proceso. El nuevo modelo de gestión de la papa incorpora los siguientes elementos:

- La organización económica de los productores y productoras con enfoque hacia la demanda, aprovechando las oportunidades del mercado mediante su segmentación y la identificación de nichos especializados.

- Generación participativa de tecnología y su integración en sistemas de manejo integrado y ecológico, con el objetivo de reducir costos, mejorar la productividad y calidad de la papa y de las prácticas de producción, mientras se mantiene o mejora la fertilidad de los suelos y del medio ambiente.

- Sistema de producción, multiplicación y comercialización de semilla de papa, centrado en pequeños productores semilleristas, para ofrecer semillas de calidad en la cantidad y oportunidad demandada.

- Acceso a las tecnologías generadas a través de la difusión y capacitación a capacitadores y capacitadoras sobre temas específicos de interés práctico de los socios del conPAPA. (Reinoso et al., 2009)

La intervención tuvo implícita una teoría del cambio (ver Figura 1). Como se observa en la figura, los factores de entrada determinantes del cambio o del logro de los impactos deseados fueron: i) aplicar un enfoque de demanda que permitió detectar las verdaderas necesidades de los pequeños productores, las cuales fueron consideradas en la planificación del proyecto, de tal forma que la intervención fue consistente y coherente para atacar directamente a los problemas o potenciar otros elementos que son parte del accionar de los productores. Las necesidades son diferentes en cada comunidad y de diverso tipo, algunas de las cuales son específicas, por ejemplo, para superar la baja productividad de los cultivos, minimizar las pérdidas por plagas y enfermedades realizando un buen manejo del cultivo; ii) lograr la participación directa y activa de los pequeños productores fue transcendental en la medida que dinamizaba el proceso de integración a los espacios de consenso y al empoderamiento.

Además se logró aplicar la metodología de las Escuelas de Campo que prácticamente consolida el proceso participativo, porque es una forma de valorar y compartir los conocimientos de los pequeños productores y por supuesto, complementar con otros conocimientos, desarrollando capacidades y habilidades en ellos, impulsando incluso la formación de capacitadores en los agricultores para que sean ellos quienes difundan el conocimiento; iii) un tercer 
Figura 1. Teoría del cambio de la intervención

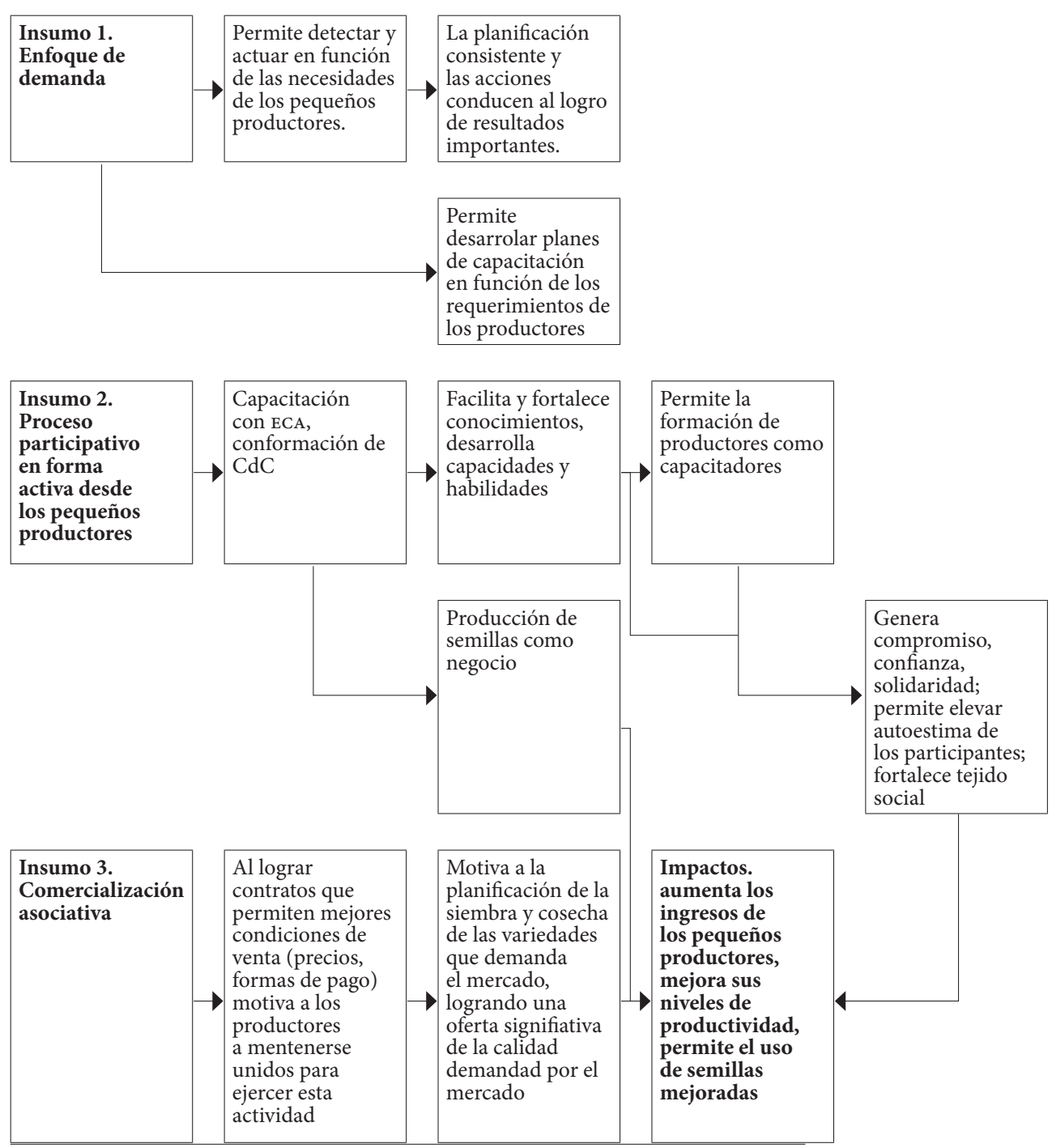

Fuente: Inferencia de la autora basada en la sistematización de la experiencia de Reinoso et al. (2009).

elemento de gran importancia para lograr el cambio fue la comercialización asociativa que les permite lograr una oferta significativa de tal forma que la negociación con los compradores se hace en el marco de una ventaja, esto es, con un poder de mercado que se reflejaba en la obtención de mejores precios y condiciones de venta en general. Dado que la negociación incluía las demandas de calidad de los compradores era necesario trabajar también en esto y así cumplir las condiciones de los contratos establecidos. Las ventas conjuntas requerían de la planificación de las siembras y cosechas a fin de que haya suficiente oferta. Es decir, para cumplir 
con los contratos de venta los productores debían poner atención a todo el proceso productivo aplicando los conocimientos adquiridos de tal forma que el producto era de buena calidad. Estos tres elementos contribuyeron a que se lograra el cambio que se ha sostenido - en particular el tercero- y que les ha permitido seguir trabajando en conjunto, encontrando nuevos compradores para los cuales han tenido que sembrar otras variedades como las nativas, contribuyendo significativamente a la agroindustria. En resumen, en el desarrollo del programa Plataformas de Concertación y CONPAPA pueden ser identificados tres etapas. La construcción de la nueva institucionalidad entre 2003 y 2005; el funcionamiento paralelo de las Plataformas y CONPAPA entre 2006 y 2007; debilitamiento de plataformas y protagonismo del CONPAPA entre 2008 y 2009. En el 2009 se concluye el financiamiento de COSUDE.

\section{DISEÑO METODOLÓGICO}

\section{EL CONTRAFACTUAL}

La clave para identificar y medir el impacto de un programa o proyecto consiste en contar con un contrafactual apropiado. Es decir, con un grupo de comparación - control- que es similar al grupo de intervención - tratamiento-, con excepción de que no ha recibido ninguna intervención. Dado que el diseño del programa Plataformas de Concertación y conPaPA no tenía previsto la evaluación del impacto, el presente estudio es un caso claro de evaluación ex post, lo que requiere de la creación de un contrafactual después de que la intervención del programa ha sido implementada. Esto implica asegurarse de que los agricultores seleccionados como grupo de control tengan características similares a las de los agricultores del grupo de tratamiento.

Para la construcción del contrafactual adecuado se utilizó el enfoque de beneficiarios versus hogares no-beneficiarios en las comunidades que no participaron en el programa. Tal enfoque descarta la inclusión al grupo de control de los agricultores que habitan en comunidades que forman parte de las áreas de intervención, aunque no son beneficiarios del programa. La decisión en este sentido fue motivada por dos reflexiones. La primera guarda relación con el hecho de que los agricultores que viven en la zona de intervención, sin ser miembros del programa, son beneficiarios indirectos de éste debido a los efectos spillover; por tanto, su inclusión en el contrafactual propicia el riesgo de subestimar el impacto total de la intervención. La segunda reflexión se refiere al hecho que la inscripción al programa tenía carácter totalmente voluntario, por lo que las personas que no se inscribieron en el programa pueden ser fundamentalmente distintas de las que se inscribieron, lo que podría conducir a un sesgo de selección en la estimación del impacto.

La elección de agricultores del grupo de control se realizó en dos pasos. En primer lugar se recopiló la información sobre los pequeños productores de papa en la provincia de Tungurahua usando los datos de la Dirección Provincial del Ministerio de Agricultura, Ganadería, Acuacultura y Pesca (MAGAP). Se identificaron zonas potenciales de los agricultores que podrían ser parte del grupo de control que tenían características básicas similares a las de las zonas tratamiento. Las particularidades utilizadas para identificar a los potenciales agricultores del grupo de control incluyeron características sociales y demográficas, de producción y tamaño de parcela. Una vez identificado un conjunto potencial de agricultores, se conversó 
con los técnicos del programa Hombro a hombro del MAGAP, que conocen bien la zona, y se pudo corroborar la selección hecha previamente.

Al mismo tiempo se diseñó un filtro adicional para mitigar el sesgo por autoselección en el grupo de control, el cual consistió en incluir una pregunta en la encuesta solo para aquéllos que nunca fueron miembros de conPaPA. Esto es, se tomó la providencia de incluir en el contrafactual solo a los agricultores que elegirían ser miembros del programa, si se presentará esta oportunidad. Con tal propósito se añadió una pregunta al cuestionario de la encuesta que mide la disposición de un agricultor a unirse a un programa de características similares - comercialización asociativa, asistencia técnica, entrega de insumos y semillas, contribución financiera mensual - considerando una escala de 1 a 5 , donde 1 refleja total desacuerdo y 5 corresponde a una alta disposición a asociarse. Los encuestados con una alta predisposición a ser parte del programa - respuestas 4 y 5 - forman parte del grupo de control.

\section{SELECCIÓN DE LA MUESTRA}

El tamaño de la muestra fue determinado en función de la potencia estadística del estudio. ${ }^{4}$ Se previó una probabilidad alta de no respuesta, por lo tanto, el tamaño inicial de la muestra se incrementó en un 50\%. La muestra contiene 92 productores del grupo de tratamiento y 89 del grupo de control. Los encuestados del grupo de control fueron seleccionados de manera aleatoria a partir del listado de los agricultores potenciales del grupo de control, elaborado con asistencia de MAGAP.

En el grupo de tratamiento se incluyeron 55 agricultores que en el año 2013 eran socios de CONPAPA, Y 37 exsocios de CONPAPA, estos últimos elegidos al azar a partir del listado proporcionado por el gerente de CONPAPA. La inclusión de los exsocios de CONPAPA al grupo tratamiento permite balancear los grupos de tratamiento y control, puesto que el grupo contrafactual incluye agricultores que se autoseleccionan para participar en un programa similar al que está bajo evaluación, pero tal participación puede ser por un corto o largo período de tiempo. A la vez, esta estrategia permite enfrentar el desgaste diferencial de la muestra (attrition).

\section{DESCRIPCIÓN DE LOS INDICADORES DE IMPACTO (VARIABLES DE RESPUESTA)}

Para determinar el éxito del programa se establecieron tres conjuntos de indicadores. El primer set, llamado indicadores primarios, mide si la intervención alcanzó su objetivo principal de mejorar el bienestar de los agricultores participantes. Si la respuesta es afirmativa significa que la intervención logró incrementar el bienestar de los participantes; el siguiente paso es considerar los mecanismos a través de los cuales se alcanzó este objetivo primario; o alternativamente, por qué la intervención pudo haber fallado en alcanzar sus objetivos. Finalmente se consideran indicadores secundarios o intermedios, que surgen de la participación en el programa (Cavatassi et al., 2011).

Los indicadores primarios seleccionados para medir si el programa logró su objetivo incrementar el bienestar de los beneficiarios- son utilidad bruta del cultivo de papa y rendimiento del cultivo, aproximado por el índice de producción. La utilidad bruta se calcula como la diferencia entre el valor total de la cosecha y los costos de producción variables totales incurridos en su producción (Berdegué Sacristan, 2001). Mientras que el índice de producción 
es el índice de producción basado en semilla, que es una medida de la productividad y se estima como la cantidad de semilla cosechada dividida para la cantidad de semilla sembrada por hectárea (Cavatassi et al., 2011). Se consideran dos aproximaciones: la productividad de la primera cosecha del año y la productividad de la cosecha total del año. Esta diferencia se hace debido que algunos agricultores anualmente realizan una sola siembra, mientras que los otros lo hacen más veces.

El programa tenía previsto mejorar el bienestar de los pequeños agricultores a través de su vinculación más efectiva a la cadena de valor de la papa. Esto es, al disminuir los costos de transacción y capturar una porción mayor del precio final para los productores. El programa también trabajó en la parte de suministro de la cadena de abastecimiento, introduciendo y abasteciendo las variedades más demandadas por el mercado, de las cuales Fripapa fue la principal. En las sesiones de las ECA se hizo hincapié en la importancia de renovar semilla de buena calidad. Adicionalmente se introdujeron diferentes y nuevas técnicas agrícolas, que en la mayoría de los casos implicó el uso adicional de insumos y, en consecuencia, costos adicionales. De este modo, los mecanismos para incrementar rendimientos probablemente incluyen una mezcla de técnicas agrícolas más efectivas combinadas con un uso mayor y más adecuado de insumos y, consecuentemente, mayores costos (Cavatassi et al., 2011). De manera general, los mecanismos de arrastre hacía el objetivo final de incrementar el bienestar de los agricultores pueden ser representados por el tiempo de transacción, el precio de venta, el costo del transporte y la aplicación de mejores técnicas agrícolas.

Finalmente se consideran indicadores secundarios para medir impactos adicionales de la participación en el programa. La introducción de nuevos paquetes tecnológicos genera dos preocupaciones. En primer lugar, como se notó en líneas anteriores, la mayoría de ellos implican el uso de fertilizantes y plaguicidas químicos, que pueden tener efectos nocivos para la salud cuando las medidas de protección no se manejan adecuadamente. En segundo lugar, la introducción de las variedades de papa altamente demandadas por el mercado, como es el caso de Fripapa.

\section{RECOLECCIÓN Y DESCRIPCIÓN DE DATOS}

Los datos fueron recolectados entre agosto y diciembre del 2013 a través de un detallado cuestionario de hogares, que incluyó varias preguntas sobre la participación en el programa. El cuestionario incluyó sets de preguntas en las siguientes áreas: i) tenencia de tierra, ii) producción de papa y uso de variedades, iii) uso de pesticidas y otros agroquímicos, iv) costos de transacción en transacciones comerciales, iv) características socio-económicas del hogar, y vi) capital social. Se realizaron varias revisiones del cuestionario durante la fase piloto, también a través de conversaciones con informantes clave, tales como el gerente de CONPAPA y el técnico de la zona del Cip.

En la encuesta participaron agricultores asentados en 18 parroquias de la provincia de Tungurahua. En nueve de ellas se ubicaban socios de CONPAPA. Se presentas datos sobre las características de los hogares de toda la muestra y de los dos grupos de control y tratamiento, es decir, hogares participantes del programa y no participantes (ver Tabla 1). La tabla presenta 
Tabla 1. Balance entre el grupo de control y el de tratamiento

\begin{tabular}{|c|c|c|c|c|}
\hline NOMBRE DE LA VARIABLE & $\begin{array}{l}\text { MUESTRA } \\
\text { TOTAL }\end{array}$ & $\begin{array}{c}\text { PARTICIPANTES } \\
\text { PROGRAMA }\end{array}$ & $\begin{array}{c}\text { NO } \\
\text { PARTICIPANTES } \\
\text { PROGRAMA }\end{array}$ & $\begin{array}{l}\operatorname{Pr}(|\mathrm{T}| \\
>|\mathrm{T}|)\end{array}$ \\
\hline \multirow[t]{2}{*}{ Años de escolaridad } & 6.02 & 5.92 & 6.11 & 0.70 \\
\hline & $(-0.24)$ & $(-0.35)$ & $(-0.33)$ & \\
\hline \multirow[t]{2}{*}{ Edad del jefe del hogar (años) } & 49.72 & 48.87 & 50.6 & 0.38 \\
\hline & $(-0.98)$ & $(-1.19)$ & $(-1.58)$ & \\
\hline \multirow[t]{2}{*}{ Pendiente de la parcela con cultivo de papas (\%) } & 14.85 & 17.14 & 12.51 & 0.01 \\
\hline & $(-0.90)$ & $(-1.29)$ & $(-1.21)$ & \\
\hline \multirow[t]{2}{*}{ Distancia de la casa a la parcela (metros) } & 801.33 & 606.20 & 1000.84 & 0.27 \\
\hline & $(-177.70)$ & $(-199.01)$ & $(-295.97)$ & \\
\hline \multirow[t]{2}{*}{ Participación en organizaciones no agrícolas (\%) } & $54.70 \%$ & $51.09 \%$ & $58.43 \%$ & 0.32 \\
\hline & $(-0.04)$ & $(-0.05)$ & $(-0.05)$ & \\
\hline \multirow[t]{2}{*}{ Participación en asociaciones agrícolas (\%) } & $4.97 \%$ & $3.26 \%$ & $6.74 \%$ & 0.28 \\
\hline & $(-0.02)$ & $(-0.02)$ & $(-0.03)$ & \\
\hline \multirow[t]{2}{*}{ Tiempo máximo en asociaciones no agrícolas (años) } & 5.4 & 5.38 & 5.42 & 0.98 \\
\hline & $(-0.68)$ & $(-0.94)$ & $(-0.99)$ & \\
\hline \multirow{2}{*}{$\begin{array}{l}\text { Número de reuniones en asociaciones no agrícolas } \\
\text { (al mes) }\end{array}$} & 0.68 & 0.293 & 1.08 & 0.13 \\
\hline & $(-0.26)$ & $(-0.19)$ & $(-0.49)$ & \\
\hline \multirow{2}{*}{$\begin{array}{l}\text { Número de reuniones en asociaciones agrícolas (al } \\
\text { mes) }\end{array}$} & 2.21 & 2.66 & 1.88 & 0.52 \\
\hline & $(-0.60)$ & $(-0.91)$ & $(-0.81)$ & \\
\hline \multirow[t]{2}{*}{ Hectáreas con cultivo de papa que tienen riego (\%) } & $50.83 \%$ & $61.96 \%$ & $39 \%$ & 0.00 \\
\hline & $(-0.04)$ & $(-0.05)$ & $(-0.05)$ & \\
\hline NÚMERO DE OBSERVACIONES & 181 & 92 & 89 & \\
\hline
\end{tabular}

Fuente: Información primaria proveniente de la encuesta levantada.

una prueba t para medias para diferentes variables, con el fin de proporcionar una evidencia inicial de la homogeneidad de los grupos de comparación.

Las pruebas de diferencias de medias muestran que el grupo de tratamiento y control son similares entre sí. Son familias donde el jefe de hogar tiene, en promedio, alrededor de 50 años de edad, con nivel educativo promedio de primaria. Viven en la misma finca. La mitad de los agricultores son miembros, en promedio por 5 años, de asociaciones no relacionadas con agricultura, tales como juntas de riego, juntas campesinas, clubes deportivos o grupos de mujeres. La participación en las asociaciones agrícolas es significativamente menor. Solo cerca del 5\% de los entrevistados pertenecen a aquellas. Sin embargo, parece que estas organizaciones demandan una participación más activa de los socios. Las reuniones en las asociaciones agrícolas se realizan cada 15 días, mientras que en las otras una vez al mes (ver Tabla 1).

Por otro lado, solo la pendiente de las parcelas y la provisión de riego son significativamente diferentes entre los dos grupos. Las fincas de los participantes del programa son más empinadas en comparación con las de no participantes (17,14\% ante 12,51\%), mientras que los participantes tienen mayor número de parcelas con riego (61,96\% ante $39 \%)$. Respecto al riego, la 
explicación está en que en muchas áreas de la provincia no lo necesitan por los altos niveles de precipitación. En definitiva, aunque parecen existir algunas diferencias entre dos grupos, las magnitudes no son grandes, y estas diferencias pueden ser fácilmente controladas en el análisis.

\section{ENFOQUE EMPIRICO}

El impacto del programa se estima con un modelo estándar de mínimos cuadrados ordinarios (мСо). Se asume que la variable de resultado $\left(Y_{i}\right)$ depende del tratamiento $D_{i}$ - donde $D_{i}=1$ si los hogares participan, 0 si no lo hacen-y de un conjunto de variables exógenas $X_{i}$, por lo tanto, el problema de la evaluación se expresa:

$Y_{i}\left(D_{i}\right)=X_{i} \beta+\alpha D_{i}+\mu_{i}(1)$

Donde $\alpha$, mide el impacto del tratamiento para el individuo $i, \beta$ define la relación entre $X_{i}$ y $Y_{i}$, mientras que $\mu_{i}$ es el término del error. Esta formulación asume que los resultados son lineales en los parámetros y que el término del error no está correlacionado con las variables exógenas $x_{i}$ y con el tratamiento.

Utilizando el grupo de comparación se trata de determinar si el programa de Plataformas de Concertación y CONPAPA fue la causa para que los pequeños productores de papa tengan mayor bienestar, lo que, por ejemplo, puede ser medido a través de una variable de resultado como la utilidad bruta $(\mathrm{UB}=\mathrm{Y})$. Es decir, para cada individuo existen dos resultados potenciales: yi(o) y yi(1). yi(o) = es la utilidad bruta de un individuo i que no es ni ha sido socio de CONPAPA, no recibió los beneficios del programa. Resultado para el contrafactual. yi $(1)=$ es la utilidad bruta (UB) de un individuo i que es o ha sido socio de CONPAPA, recibió los beneficios del programa.

\section{RESULTADO PARA EL GRUPO DE TRATAMIENTO}

Cada individuo i puede escoger una de las dos opciones alternativas: participar en el programa o no - ser socio o no-; por lo tanto, tiene dos resultados potenciales respecto a la utilidad bruta obtenida. Evidentemente, para cada individuo solo uno de estos resultados podrá ser observado. Si denominamos $\mathrm{D}$ al estado que el individuo escoge: $\mathrm{D}=1$ si participa en CoNPAPA y D=o si no participa; yi(o) será el resultado potencial de la utilidad bruta si no participa, y yi(1) si participa. Es decir:

$Y_{i}(D)=Y_{i}(0)\left(1-D_{i}\right)+Y_{i}(1) D_{i}$

Donde:

$$
\begin{array}{lc}
Y_{i}(0)=X_{i} \beta_{0}+\mu_{i 0} & E\left(\mu_{i 0} \mid X_{i}\right)=0 \\
Y_{i}(1)=X_{i} \beta_{1}+\mu_{i 1} & E\left(\mu_{i 1} \mid X_{i}\right)=0
\end{array}
$$

Reemplazando las dos ecuaciones anteriores en la primera obtenemos:

$\Delta_{i}=Y_{i}(1)-Y_{i}(0)$ 
Tabla 2. Estimación de MCo de los efectos del tratamiento

\begin{tabular}{|c|c|c|c|c|}
\hline \multirow[t]{2}{*}{ VARIABLES DE RESULTADOS } & \multicolumn{2}{|c|}{$\begin{array}{l}\text { SIN VARIABLES } \\
\text { DE CONTROL }\end{array}$} & \multicolumn{2}{|c|}{$\begin{array}{l}\text { CON VARIABLES } \\
\text { DE CONTROL }\end{array}$} \\
\hline & DIFERENC. & ERROR EST & DIFERENC. & ERROR EST. \\
\hline \multicolumn{5}{|l|}{ INDICADORES PRIMARIOS } \\
\hline Îndice de producción, total cosecha año & 0.489 & $(0.593)$ & 0.574 & $(0.575)$ \\
\hline Índice de producción, primera cosecha año & 1.092 & $(0.692)$ & $1.260^{*}$ & $(0.665)$ \\
\hline Utilidad bruta (USD/ha) & 754.45 & $(466.32)$ & $1121.01^{* *}$ & $(420.80)$ \\
\hline Utilidad bruta primera cosecha (USD/ha) & $1703^{* * *}$ & $(538.7)$ & $1892.26^{* * *}$ & $(489.30)$ \\
\hline \multicolumn{5}{|l|}{ INDICADORES DE MECANISMOS } \\
\hline Costo de transporte (USD $/ \mathrm{kg}$ ) & $-0.005^{* * *}$ & $(0.0008)$ & $-0.006^{* * *}$ & $(0.0008)$ \\
\hline Tiempo de transacción (horas) & $-1.321^{* *}$ & $(0.543)$ & $-1.397^{\star \star}$ & $(0.621)$ \\
\hline Precio de venta de las papas (usd/qq) & $3.119^{* * *}$ & $(0.481)$ & $2.878^{* * *}$ & $(0.489)$ \\
\hline Costo de los insumos (USD/ha) & $319.5^{* * *}$ & $(84.10)$ & $347.9^{* * *}$ & $(91.17)$ \\
\hline Costo de insumos primera siembra (USD/ha) & $270.4^{* * *}$ & $(86.59)$ & $230.3^{* * *}$ & $(80.07)$ \\
\hline Costo de insumos sin fertilizante primera siembra (USD/ha) & $253.3^{* * *}$ & $(83.73)$ & $220.7^{* * *}$ & $(78.13)$ \\
\hline Cantidad de semillas compradas (kg/ha) & $340.4^{\star \star}$ & $(126.2)$ & 240.8 & $(149.10)$ \\
\hline Cantidad de semilla comprada primera siembra (kg/ha) & $343.5^{\star *}$ & $(138.7)$ & 133.1 & $(172.7)$ \\
\hline$\%$ de semilla comprada respecto al total de semilla usada & $0.195^{\star * *}$ & $(0.059)$ & 0.0924 & $(0.0642)$ \\
\hline $\begin{array}{l}\text { \% de semilla comprada respecto al total de semilla usada en la } \\
\text { primera siembra }\end{array}$ & $0.244^{* * *}$ & $(0.0636)$ & $0.128^{*}$ & $(0.0678)$ \\
\hline Costo de la semilla comprada (usD/ha) & $167.0^{* * *}$ & $(38.68)$ & $146.04^{\star * \star}$ & $(42.11)$ \\
\hline Costo de la semilla comprada primera siembra (USD/ha) & & & & $(22.47)$ \\
\hline Costo de los fertilizantes químicos (USD/ha) & $80.58^{* *}$ & $(36.76)$ & $98.70^{* * *}$ & $(36.51)$ \\
\hline Costo de los fertilizantes químicos primera siembra (USD/ha) & $84.98^{\star *}$ & $(37.64)$ & $69.61^{* *}$ & $(33.35)$ \\
\hline Calificación del conocimiento de la «lancha» & $1.089^{\star * *}$ & $(0.306)$ & $0.939^{* * *}$ & $(0.331)$ \\
\hline Calificación del conocimiento del «gusano blanco» & $1.073^{* * *}$ & $(0.291)$ & $0.963^{* * *}$ & $(0.312)$ \\
\hline Calificación del conocimiento de la "polilla» & $1.134^{* * *}$ & $(0.338)$ & $0.922^{* * *}$ & $(0.354)$ \\
\hline \multicolumn{5}{|l|}{ INDICADORES SECUNDARIOS } \\
\hline Número de variedades sembradas & $0.349^{* * *}$ & $(0.132)$ & 0.165 & $(0.147)$ \\
\hline Uso de algún tipo de protección & 0.0867 & $(0.073)$ & 0.0744 & $(0.076)$ \\
\hline Uso del poncho plástico & $0.178^{* * *}$ & $(0.0635)$ & $0.159^{* *}$ & $(0.0695)$ \\
\hline Uso de máscara & 0.0297 & $(0.0508)$ & 0.0212 & $(0.0538)$ \\
\hline Primera variedad de papa más usada (superchola) & 0.0409 & $(0.0713)$ & 0.0353 & $(0.0747)$ \\
\hline Segunda variedad de papa más usada (Fripapa) & 0.0840 & $(0.0588)$ & 0.0744 & $(0.0552)$ \\
\hline Tercera variedad más usada es (Gabriela) & $-0.171^{\star * *}$ & $(0.0472)$ & $-0.124^{* *}$ & $(0.0493)$ \\
\hline Calificación de la identificación del producto más tóxico & $0.472^{* *}$ & $(0.202)$ & $0.409^{*}$ & $(0.212)$ \\
\hline NÚMERO DE OBSERVACIONES & 181 & & & \\
\hline
\end{tabular}


Una cuidadosa selección de los individuos para los grupos de tratamiento y de control, nos asegura el control de los inobservables que pueden haber llevado a algunos hogares a unirse al programa. De esta manera, cualquier sesgo asociado a la autoselección es eliminado. Por lo tanto, el hecho de que el término de error no esté correlacionado con el tratamiento es una suposición razonable. Por otro lado, existen diferencias en las variables exógenas entre estos hogares (ver Tabla 1), pero la magnitud es pequeña. La inclusión de este set de variables al modelo como variables de control, permite estimar el impacto del programa de manera insesgada.

Cabe señalar que como el grupo de control está conformado por miembros — supervivientes- y exmiembros y proporción de los supervivientes no es representativa, se utiliza ponderadores muestrales que representan la inversa de la probabilidad de ser seleccionados, de este modo se pueden tener estimadores insesgados y consistentes (Solon, Haider and Wooldridge, 2013).

\section{DISCUSION DE LOS RESULTADOS}

Los resultados de estimación de impactos (ver Tabla 2) muestran que los dos indicadores primarios de impacto están positiva y significativamente influenciados por la participación en el programa. Los resultados indican que los índices de producción son $26 \%$ más altos para participantes del programa y las utilidades brutas por hectárea son mayores en USD 1892,26 cuando se tiene la única cosecha al año y USD 1121,01 más grandes cuando se siembra varias veces.

Los mecanismos empleados para alcanzar estos resultados muestran que los beneficiarios pueden vender más de su cosecha en comparación con los no beneficiarios, debido al incremento en la cantidad cosechada por hectárea. La cantidad vendida también proporcionó un mayor valor a los vendedores beneficiarios, lo cual es posible a través de un precio significativamente más alto. Los precios obtenidos por un quintal de papa fueron alrededor de USD 3,00 más altos que aquellos que no participaron en el programa. Sin embargo, los costos de los insumos también son más altos para los beneficiarios, lo que se debe principalmente a costos significativamente mayores de la semilla sembrada, insumos en general y fertilizantes químicos en particular. Todo esto puede ser relacionado con los nuevos paquetes tecnológicos que los participantes adquirieron siendo parte del programa.

El incremento en la productividad también encuentra su explicación a través del manejo adecuado del material de siembra. Los beneficiarios del programa renuevan la semilla por lo menos en la primera siembra del año, más frecuentemente que los no beneficiarios. El porcentaje de semilla comprada es $12,8 \%$ mayor entre los participantes del programa que entre los no participantes. Los resultados muestran que los beneficiarios del programa reconocen con mayor facilidad las plagas y enfermedades que atacan al cultivo de la papa -lancha, gusano blanco, polilla-, lo que les permite realizar un control más efectivo de los sembríos. Esto, sin duda, tiene un efecto positivo sobre los rendimientos y, al mismo tiempo, puede influir para alcanzar un precio más alto, que normalmente es como un premio a una mejor calidad del producto.

La mejora de la calidad del producto parece ser un determinante para evadir la cadena de intermediarios de las ferias mayoristas y vincularse al mercado de manera más directa. Los 
menores tiempos de transacción y costos de transporte, que enfrentan los beneficiarios del programa apoyan esta idea. Los dos indicadores muestran valores negativos significativos, $-1,397$ horas por transacción y -0,006 dólares por kilogramo de papa, respectivamente. En conjunto, parece que mientras los agricultores beneficiarios pagaron más por algunos insumos claves, recibieron los beneficios de la inversión a través de mayores rendimientos y mayores precios y, así, mayores ganancias en la producción de papa.

Pasando a los indicadores secundarios de impacto, existe alguna preocupación respecto a que la vinculación de los pequeños productores al mercado pueda llevar a mayores ganancias a costa de mayores problemas ambientales y de salud. Respecto al primer problema, los beneficiarios del programa no muestran una diferencia significativa en el número de variedades sembradas frente a los no beneficiarios, pues en general la lógica campesina es la diversificación de cultivos y variedades en la parcela. Tampoco se evidencia la diferencia en sembríos de la variedad introducida de Fripapa. Tanto en las fincas de los participantes como de no participantes, Fripapa ocupa el segundo lugar en los cultivos de papa.

Por otro lado, la preocupación en torno a los efectos nocivos en la salud de los agricultores debido a las tecnologías introducidas tiene una base objetiva. A pesar de las evidencias de que los beneficiarios del programa utilizan mayor cantidad de químicos, no se diferencian de los no beneficiarios en el uso de equipos de protección en general y de mascarillas en particular, a excepción de los ponchos de plástico. Sin embargo, los beneficiarios cuentan con mayor capacidad de distinguir el grado de toxicidad de los químicos. Si bien este hecho no aboga a favor de la idea que los beneficiarios utilizan productos químicos de menor toxicidad, sí evidencia que las enseñanzas de las ECA perduran en el tiempo.

\section{CONCLUSIONES}

En este documento se examina el impacto de Plataformas de Concertación y conPAPA, programa que se inscribe dentro del paradigma del desarrollo agrícola como un medio de combatir la pobreza en el sector rural. El objetivo del programa consiste en mejorar el bienestar de los pequeños productores de papa en la Sierra central del Ecuador. Para cumplir con tal propósito, el programa activa una serie de mecanismos orientados a vincular a los pequeños agricultores al mercado de forma más efectiva. En este sentido, las acciones emprendidas por el programa pueden ser calificadas como medidas para el incremento de la productividad, lo que permite ofertar mayor cantidad del producto en el mercado y medidas para el incremento de precio de la papa ofertada.

Se propone lograr el incremento de la productividad de la finca campesina mediante la introducción de nuevas variedades de papa demandadas en el mercado, lo que implica la asimilación por parte de los beneficiarios de los paquetes tecnológicos con el uso más intensivo de agroquímicos. Por otro lado, se apuesta a la transmisión del conocimiento que permite un manejo integral del cultivo de papa. En cuanto al incremento del precio se apunta a acortar la cadena de valor de la papa de tal manera que el agricultor podría apropiarse de la parte del valor tradicionalmente retenido por el intermediario; un efecto adicional de esta acción se da cuando disminuyen los costos de transacción y del transporte. 
Para evaluar el impacto del programa se adaptó un conjunto de indicadores en tres niveles. El primer nivel, los indicadores primarios, se utilizó para medir el alcance del objetivo del programa, entendido este como el incremento del bienestar de los pequeños productores. Con tal propósito se utilizaron los índices de rendimientos y márgenes de ganancia. El segundo nivel de análisis incluyó la valoración de los mecanismos que utilizó el programa para alcanzar el objetivo primario. Entre estos se consideraron costos de transacción y de transporte, precio de venta de papa y las técnicas agrícolas adoptadas. El tercer nivel lo consolidaron indicadores secundarios, que permitieron evaluar los otros impactos del programa, específicamente el impacto ambiental y a la salud de agricultores.

Con el fin de asegurar la identificación del impacto del programa se construyó un contrafactual razonable para comparar a los beneficiarios, y se utilizó el método de Mco. Un elemento clave que ha ayudado a controlar un potencial sesgo por autoselección fue la selección adecuada de los participantes del grupo de tratamiento y de control. El grupo de tratamiento fue conformado por individuos que pertenecen a la sociedad de los agricultores CONPAPA y otros que fueron sus socios en algún momento. El grupo control está constituido por agricultores que habitan en las zonas no intervenidas por el programa, pero que están dispuestos a ser su partícipe si se presenta una oportunidad para esto.

Los resultados de la evaluación apuntan a que el programa incrementó con éxito el bienestar de los agricultores beneficiarios. Todos los impactos relacionados con los objetivos primarios de la plataforma, que son rendimientos y utilidades brutas, son positivos y están influenciados significativamente por la participación en el programa. Los mecanismos a través de los cuales el programa alcanza este éxito son la reducción y aumento de la eficiencia de la cadena de valor de la papa, así como mediante la aplicación de mejores técnicas agrícolas, reduciendo los costos de transacción con el primero y mejorando rendimientos con el segundo. A pesar de que el programa tuvo un efecto positivo sobre los rendimientos del cultivo de papas, alcanzándose una producción promedio de 10,3 t/ha en las parcelas de los participantes, un rendimiento mayor que el promedio provincial $-7,3 \mathrm{t} / \mathrm{ha}-\mathrm{y}$ nacional $-8,3 \mathrm{t} / \mathrm{ha}-$, la productividad de los cultivos de los beneficiarios sigue siendo sustancialmente más baja que el rendimiento promedio en América Latina (17,6 t/ha) (INEC, 2013).

Por último, los impactos secundarios relacionados principalmente con el medioambiente y la salud, muestran que no hay evidencias que el programa fomente la reducción de la biodiversidad de la finca a través de sustitución de las variedades nativas por las demandadas por el mercado. Sin embargo, los impactos a la salud que genera el uso de agroquímicos evocan preocupación, debido al desuso de los equipos de protección.

El análisis presentado tiene especial relevancia en vista de que los datos fueron recolectados en el 2013, cuatro años después de retirado el apoyo financiero al programa por la cosudE, cuando hay productores que se mantienen como miembros y otros no. El hecho de que los impactos positivos observados perduren en el tiempo, proporciona evidencias de que el programa activó los mecanismos idóneos para vincular al pequeño productor al mercado con el consecuente incremento de sus ingresos. Los impactos positivos del programa, identificados por Cavatassi en la investigación de 2008, siguen presentes, a excepción del uso de los equipos de protección por parte de los agricultores, que mostró una degeneración con el tiempo. 


\section{NOTAS}

1 Gobierno Autónomo Descentralizado: gobiernos municipales, provinciales o parroquiales. 2 Solo por citar algunos actores: los GAD como actores principales de la planificación del desarrollo local incluirían como parte de esta a las organizaciones de pequeños productores. En el eslabón de la producción, el Centro Internacional de la Papa (CIP) e Instituto Nacional de Investigaciones Agropecuarias (INIAP), como entes con amplio conocimiento en el manejo del cultivo, aportarían impulsando proyectos de investigación en tecnologías y variedades de papas para el mercado. En lo que se relaciona a organización de productores apoyaban actores locales como Fundación Marco, Ayuda en Acción, proyecto CIERch de la Diócesis de Riobamba y los grupos de agricultores.

3 Se dice que los mercados de productos de alto valor son de productos procesados o a los que se les agregado valor. Por ejemplo, no es lo mismo vender trigo que harina de trigo, donde se obtienen 6,7 veces más ingresos, aunque la molienda no es una actividad de alto procesamiento según el IICA (1991).

4 Coeficiente de poder del 9o\%, confianza de confianza del 95\%; el efecto mínimo, estimado a partir de los datos del estudio de Catavassi et al., (2009).

\section{REFERENCIAS}

Andrade Piedra, J., Kromannr, P., Taipe, A., Devaux, A., Forbes, G., Flores, P. y Ortiz, O. (2013). Primera aproximación a la estrategia del centro internacional de la papa para mejorar la calidad de la semilla y facilitar el acceso a nuevas variedades. En v Congreso de la Papa, Memorias: v Congreso ecuatoriano de la papa. IV Congreso Iberoamericano sobre la investigación y desarrollo de la papa. III feria Expopapa (pp. 68-69). Riobamba, Ecuador: INIAP-CIP ESPOCH-MAGAP.

Bachke, M. E. (2009, abril). Are farmers' organizations a good tool to improve small-scale farmers' welfare? II Conferencia do IESE, Dinamicas da pobreza e padrões de acumulação. Maputo, Moçambique.

Banco Mundial. (2008). Informe sobre el desarrollo mundial 20o8: Agricultura para el desarrollo. Washington, DC, UsA: Banco Mundial/Mayol Ediciones s. A.

Berdegué Sacristán, J. A. (2001). Cooperating to compete. Associative peasant business firms in Chile. (Doctorate thesis). Wageningen University. Güeldres, Netherlands.

Bernard, T., Taffesse, Alemayehu Seyoum, A. and Gabre-Madhin, E. (2008). Impact of cooperatives on smallholders' commercialization behavior: evidence from Ethiopia. Agricultural Economics, 39, 147-161.

Camagni, M. y Kherallah, M. (2016, enero 16). Proyectos de desarrollo de cadenas de valor de productos básicos: Inclusión sostenible de pequeños productores en cadenas de valor agrícolas. Fondo internacional de desarrollo agrícola, FIDA. Recuperado de https://www.ifad.org/ documents/1018o/656f769d-7017-4e1e-9c45-72ccfbb5ab4f

Cavatassi, R., González, M., Winters, P., Andrade, P. J., Thiele, G. and Espinosa, P. (julio 2011). Linking smallholders to the new agricultural economy: An evaluation of the Plataformas program in Ecuador. Journal of Development Studies, (s. d.), 1-29,

Cavatassi, R., González, M., Winters, P., Andrade-Piedra, J., Thiele, G. and Espinosa, P. (2011). Linking smallholders to the new agricultural economy: An evaluation of the Plataformas program in Ecuador. Journal of Development Studies, 1-29.

Crespo Coello, P., Guerrero, D., Iturralde, P., Mera, X., Monteros, C., Montesdeoca, F. y Yumisaca, F. (2005). Por una nueva institucionalidad de la papa en el Ecuador. Quito, Ecuador: INIAP-COsUdE.

FAO. (2011, febrero 28). Ecuador en una mirada. FAO. Recuperado de http://www.fao.org/ecuador/ fao-en-ecuador/ecuador-en-una-mirada/es/ 
Fischer, E. and Qaim, M. (2012). Linking smallholders to markets: determinants and impacts of farmer collective action in Kenya. World Development, 40, 1255-1268.

Godtland, E., Sadoulet, E., De Janvry, A., Murgai, R. and Ortiz, O. (2004). The impact of farmer field schools on knowledge and productivity: a study of potato farmers in the Peruvian Andes. Economic development and cultural change, 63, pag. 63-92.

González-Flores, M., Bravo-Ureta, B. E., Solís, D. and Winters, P. (2014). The impact of high value markets on smallholder productivity in the Ecuadorean Sierra: A Stochastic Production Frontier approach correcting for selectivity bias. Food Policy, 44, pag. 237-247.

IICA. (1991). La agricultura en América Latina y el Caribe: Estrategias para fin de siglo. X Conferencia Interamericana de Ministros de Agricultura. Madrid, España.

INEC. (2013). Informe ejecutivo: Encuesta de superficie y producción agropecuaria continua. Quito, Ecuador: INEC.

INEC. (2014). Pobreza (junio 2014). Quito, Ecuador: INEC.

Pumisacho, M. (2006, mayo). Escuelas de campo, base para la construcción de plataformas de concertación, experiencia desarrollada por el PNRT-papa del INIAP. Instituto Nacional de Investigaciones Agropecuarias. Recuperado de http://repositorio.iniap.gob.ec/handle/4100o/4402

Razeto M., L. (1999). La Economía Solidaria: Concepto, realidad y proyecto. Persona y Sociedad, (13)2, (s. d.).

Reinoso, I., Andrade Piedra, J., Pico, H., Motesdeoca, F., Monteros, C., Pumisacho, M. y Yumisa, F. (2009). Somos paperos. Cambios que sirven: La experiencia de las plataformas y del CONPAPA. Quito, Ecuador: CIP-COSUDE.

SENPlades. (2010). Agenda Zonal para el Buen Vivir. Propuestas para el desarrollo y lineamientos para el ordenamiento territorial: Zona de planificación 3, provincias de Chimborazo, Cotopaxi, Tungurahua y Pastaza. Secretaría Nacional de Planificación y Desarrollo. Recuperado de http:// www.planificacion.gob.ec/wp-content/uploads/downloads/2012/o8/Agenda-zona-3.pdf

Senplades. (2015). Agenda Zonal: Zona-3 Centro. Cotopaxi, Cimborazo, Tungurahua y Pastaza. 2013-2017. Secretaría Nacional de Planificación y Desarrollo Recuperado de http://www.planificacion.gob. ec/wp-content/uploads/downloads/2015/11/Agenda-zona-3.pdf

Sikwela, M. and Mushunje, A. (2013). The impact of farmer support programmes on household income and sustainability in smallholder production: A case study of the Eastern Cape and KwaZulu Natal farmers, South Africa. African Journal of Agricultural Research, (8)21, 2502-2511.

Solon, G., Haider, S. J. and Wooldridge, J. (2013, February). What are we weighting for. National Bureau of Economic Research (s. d.). Recuperado de http://www.nber.org/papers/w18859

Vollrath, T. (1994). The role of agriculture and its prerequisites in economic development. Food Policy, (19)5, 473.

Wainaina, P., Okello, J. and Nzuma, J. (2012, august). Impact of contract farming on smallholder poultry farmers' income in Kenya. Selected paper prepared for presentation at the International Association of Agricultural Economists (IAAE) Triennial Conference. Foz do Iguaçu, Brazil. 